\title{
Artificial Intelligence - Creating the Future in Orthodontics - A Review
}

\author{
Jeyaram Palanivel ${ }^{1}$, Davis D. ${ }^{2}$, Dilip Srinivasan ${ }^{3}$, Sushil Chakravarthi N.C.4, \\ Priya Kalidass ${ }^{5}$, Shreya Kishore ${ }^{6}$, Suvetha S.7 \\ 1, 2, 3, 4, 5, 6, 7 Department of Orthodontics, SRM Dental College, Ramapuram, Chennai, Tamil Nadu, India.
}

\section{ABSTRACT}

With the search for a smarter, faster, and technological ways of getting things accomplished, Artificial Intelligence (AI) is developing at a faster pace. The technology has become a part of daily life, where the blend of human intelligence and machine learning has reached heights in various fields of science and technology. The machine simulates the human intelligence and improves their abilities with the help of self-adapting algorithms.

Artificial intelligence has provided many benefits in various fields, particularly in medicine, where it plays a major role in the advancement of the medical field, ranging from virtual assistants to creating a better diagnosis and treatment using accumulated patient data. In orthodontics, the treatment focuses on altering the occlusion, controlling the development of dentoalveolar components and growth abnormalities. An effective assessment of these problems enables in determining the need for treatment and to prioritize it. Precise diagnosis, offering relevant and complete information is a key to a successful practice in orthodontics. Of late artificial intelligence is applied in orthodontics in decision making and planning effective treatment outcomes.

Artificial intelligence is useful in simulation of various clinical scenarios in the three-essential sequence - diagnosis, treatment planning and treatment, which is efficient enough in reducing the workload, time and also increases the accuracy and monitoring. In no ways artificial intelligence can replace the dentist because clinical practice is not just about the diagnosis and treatment plan. So, humans should have a basic understanding on artificial intelligence models to assist in clinical judgement and not to replace the knowledge and expertise of humans.

\section{KEY WORDS}

Artificial intelligence, Machine Learning, Artificial Neural Network, Orthodontics, Review
Corresponding Author:

Dr. Jeyaram $P$.

111/66/11C, S. K. S. R Colony,

Ezhil Nagar West,

Tuticorin - 628002,

E-mail: jeyaram1296@gmail.com

DOI: $10.14260 / j e m d s / 2021 / 431$

How to Cite This Article:

Palanivel J, D. David, Srinivasan D, et al. Artificial intelligence - creating the future in orthodontics - a review. J Evolution Med Dent Sci 2021;10(28):2108-2113, DOI: $10.14260 / \mathrm{jemds} / 2021 / 431$

Submission 09-03-2021,

Peer Review 12-05-2021,

Acceptance 19-05-2021,

Published 12-07-2021.

Copyright (c) 2021 Jeyaram Palanivel et al. This is an open access article distributed under Creative Commons Attribution License [Attribution 4.0 International (CC BY 4.0)] 


\section{BACKGROUND}

Artificial intelligence (AI) is prevalent in various fields of daily life. Currently, AI based algorithms are involved in everyday technology and are being extensively used in search engines of internet, online assistance, and face recognition in various social media platforms. In general, artificial intelligence is described as "computers mimicking human intelligence". Specifically, machine learning, as a category of artificial intelligence, is characterized by mathematical and statistical techniques enabling machines to improve their abilities by using self-adapting algorithms. ${ }^{1}$

The apt definition for AI calls for formalization of the term intelligence. Intelligence aids in recognizing the right piece of knowledge at appropriate instance of decision making. Artificial intelligence can be defined as the simulation of human intelligence on a machine. AI is based on disciplines from philosophy, psychology, computer science, mathematics, and engineering. All these contribute in building up an intelligent system. Artificial intelligence encompasses a wider horizon. It deals with a variety of information representation schemes, intelligent search, methods for solving uncertainty of data and knowledge, different ideas for automated machine learning and much more. Expert systems, image recognition, game - playing, theorem - proving, language processing and robotics are also the few areas of application of $\mathrm{AI}^{2}{ }^{2}$ With recent advancements in computing,

AI algorithms can be used for abstract and complex tasks. They are emerging as a promising field in health care. AI algorithms assist clinicians to analyse medical imaging, diagnose diseases and to support therapeutic decisions. ${ }^{1} \mathrm{AI}$ is being applied in the field of medicine like the online scheduling of appointments, online check-ins, digitalization of records, reminder for follow up and immunization and warning of adverse effects while prescribing multidrug combinations. In orthodontics, the treatment focuses on altering the occlusion, controlling development of dentoalveolar components and growth abnormalities. An effective assessment of problem enables in determining the treatment need and priority.

As the medical care demanded more monetary, discovery of computer and its use in the field of medicine gained more interest, which paved the way for introduction of artificial intelligence. ${ }^{2} \mathrm{Offering}$ precise and complete information prior to the start of the treatment is very crucial in orthodontics. Orthodontists need to notify patients about the treatment plan, alternative methods, duration of the treatment, cost, extent of expected success and potential risks during the consultation stages. With the help of patient's medical history, $\mathrm{x}$-rays of the head and teeth and the intra oral and extra oral examination are done to arrive at the accurate diagnosis. Using a computerized intelligent model that could suggest the best treatment protocol and forecast the degree of the treatment success would be a great consulting resource. ${ }^{3}$ This article focuses on the association between artificial intelligence and orthodontics.

\section{ARTIFICIAL NEURAL NETWORKS}

An Artificial Neural Network (ANN) is a computational or mathematical model centred on the biological signal of the human brain. It operates parallel, assuming that computation is distributed over the interconnected processing elements called neurons or nodes. An ANN model is built on structural learning and analysis, mechanism, and functions of the biological neural networks. ${ }^{3,4}$ The basic unit of the neural network is the neuron, which obtains signal through the synapses situated on the dendrite or the neuronal membrane. The signal received is effective enough to activate the neurons and radiate the signals through the axon, which in turn activates the other neurons by another synapse. ${ }^{2}$

The neural network regulates centred on the comparison of the target and the output, until the output of the network matches the target. Various input / target pairs are required to train the network, and these are termed as Artificial Neural Networks. The structural design is the arrangement of neurons into layers and the connection between them, which is associated with learning algorithms. The output nodes of one layer are transferred to the other layer by connections that amplifies or attenuates the outputs through weight factors. The net input of each node is the sum of outputs of the nodes in the previous layers, except for nodes on the input layer. The nodes fire an output when they are activated in harmony with input, activation function and threshold of the node. ${ }^{3}$ (Fig 1)
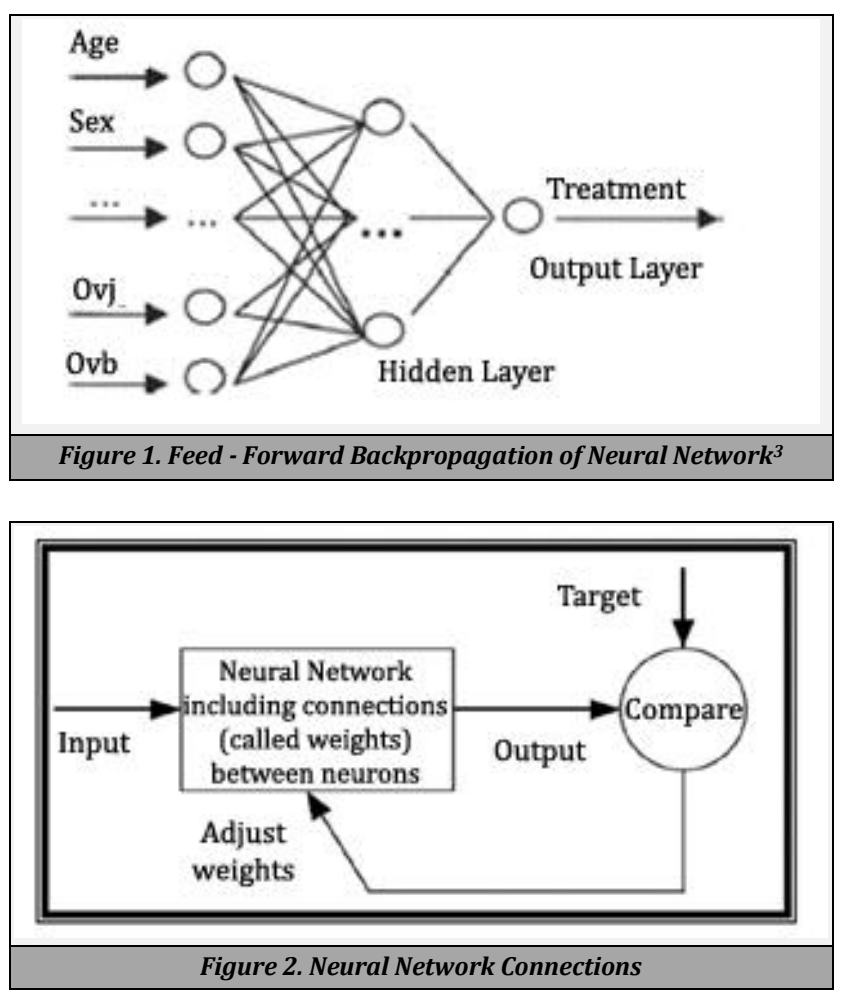

ANN can process non-linear relationship and exhibit learning ability. It offers researchers with advantages like large scale parallelism, distribution representation of knowledge, robustness and self-organization that deals as a new approach to complex problems. 
The ANN models are of benefit in the medical research as they can process complicated uncertain problems, nonconfiguration, non- linearity and multiple factor interactions to resolve prognostication, classification, pattern recognition and image processing. ${ }^{4}$ (Fig 2)

\section{Benefits of Using Neural Network}

1. Nonlinearity - Artificial neurons are of two types - linear and non-linear. A linear neuron can be modelled by nonlinear neurons but the opposite cannot be done.

2. Input and output mapping - The network creates an input - output mapping for the problem from the previous samples which are organized in different manner. The conventional learning process of neural network is performed supervised learning.

3. Adaptivity - The neural networks adapt the synapses to the surrounding environmental changes by retraining the model or making the changes in the network. It is useful for pattern classification, processing of signals and application control.

4. Fault tolerance - The neural network implemented in hardware form could be innately fault tolerant or capable of robust control. ${ }^{2}$

\section{Classification of Machine Learning (ML) Algorithms}

ML algorithms are categorized depending on the nature of learning and desired outcome of the algorithm:

1. Supervised learning

2. Unsupervised learning

3. Reinforcement learning

\section{Supervised Learning}

The data can be discrete or continuous. The supervised learning uses the data for classification if it is discrete and for prediction if it is continuous. The name supervised learning came because of the known outcome.

There are two types of variables; dependent and independent in which set of training data is independent variable and known outcome is dependent and the system confirms or rejects the performance based on the feedback signals. In orthodontics, the most commonly used ML algorithm is supervised learning as it provides clinical decision on orthodontic extractions where the Neural Network learning is built based on clinical examination, orthodontic casts, and radiographic measurements.

\section{Unsupervised Learning}

In this, the data is analysed and classified into groups based on clustering or unsupervised classification. This learning is helpful in organizing the data to get a significant information. Here the data is not labelled, and the final outcome is not known.

\section{Reinforcement Learning}

It is similar to supervised learning but the only difference is the feedback signal which rewards the system instead of adding value. Here the system has no knowledge on the environmental behaviour and by the multiple exploratory trial and error, it learns to enhance its future performance. ${ }^{5}$

\section{APPLICATIONS OF AI TECHNIQUES}

Few of the applications are

1. Expert systems - Comprises of a knowledge base, database and an inference engine to interpret the database using the knowledge provided.

2. Image understanding and computer vision - Useful in receiving the illumination from the image $(2 \mathrm{D}$ array of pixels)

3. Navigational Planning for mobile robots - Also called automated guided vehicles (AGV). The issue in navigation planning endures in both static and dynamic environments.

4. Speech understanding - The determined features like amplitude, fundamental and harmonic frequencies of each syllable are present in a spoken word and the issue is to separate the syllables which are recognized by pattern classification technique.

5. Scheduling - For effective management of time, the set of events are organized in time schedule.

6. Intelligent control - As per the objectives, the controller is formed from the renowned model of the process.

7. System modelling and optimization - Optimization methods are being used from a very long time to create solutions that solely maximizes the performance. ${ }^{2}$

\section{Artificial Intelligence in Medicine}

An important branch in the field of $\mathrm{AI}$ is the Expert system (ES), which is computer program system that processes knowledge and information comprising of knowledge base and inference machine. It mimics making of decisions and the working processes of the experts and resolves the actual problems in the field of a single specialty. ${ }^{2}$

\section{Artificial Intelligence in Dentistry}

AI has great ability in recognizing meaningful data and is being widely used for clinical trials, decision making in diagnosis, treatment, and prognosis phase. In dentistry it is slowly gaining attention in the field of radiology with more importance to diagnostic records with three dimensional scans and CBCT to help in faster diagnosis and plan the treatment. The most talked about AI being used recently is in the field of orthodontics for the customized orthodontic treatment. Poedijasteti $\mathrm{W}$ et al. investigated the application of AI by taking panoramic radiographs to diagnose a lesion or a tumour in which $83 \%$ of the cases were precisely diagnosed as ameloblastoma and keratocystic odontogenic tumors. ${ }^{6}$ Yong et al. developed a machine learning to successfully predict odontogenic cysts, dentigerous cysts, osteomyelitis, periapical cysts and ameloblastoma which was correct $90.6 \%$, $90.9 \%, 99.4 \%, 89.6 \%$, and $100 \%$ of the time on an average of 38 seconds. It was also viewed by five oral and maxillofacial surgeons who examined radiographs and diagnosed the problems $82.9 \%$ of the time on an average of $23.1 \mathrm{~min}$ for each diagnosis. ${ }^{7}$ Yilmaz et al. developed an automated system utilizing support vector machine to identify periapical cyst and keratocystic odontogenic tumours all the time using cone beam computed tomography (CBCT). ${ }^{8}$ 
ARTIFICIAL INTELLIGENCE IN ORTHODONTICS

\section{In Diagnosis}

\section{Cephalometric Radiology}

Few of the applications are automated diagnosis, data mining and detection of landmarks. The major drawback in manual cephalometry is identification of landmarks. The diagnostic value of analysis depends on the accuracy and reproducibility of landmark identification. The developing computational power and algorithms lead to improvement in accuracy, reliability, and efficiency. ${ }^{9}$ Arik et al. used conventional neural networks to diagnose from cephalograms. The results of the study concluded that there was $5-15 \%$ better accuracy of detecting landmarks with AI than traditional methods. ${ }^{10,11}$

Digital cephalometric analysis improved the diagnosis by reduction of errors and saving time. Lee et al. assessed the analysis of cephalometric radiographs using deep convolutional neural networks (DCNN for the differential diagnosis of the indications of orthognathic surgery. With this DCNN speed increased and complex calculations could be performed in a shorter time. ${ }^{12}$

Park et al. compared two different algorithms - YOLO version 3 (YOLOv3) and Single Shot Multibox Detector (SSD) to identify 80 landmarks. YOLOv3 was better than SSD in terms of precision, computational time and detected more errors than SSD. 9,13

Leonardia $\mathrm{R}$ et al. cited the different approaches investigated by various authors into different groups.

1. Image filtering plus knowledge - based landmark search

- Easy to implement, better accuracy and proper anatomical knowledge

- Fail to capture the morphological variability

- Highly dependent on image quality, intensity level

2. Model - based approaches

- Invariant to scale, rotation, translation and shape variability

- Not always precise

- Sensitive to noise

3. Soft - computing approaches

- Accommodates shape variability

- Tolerant to noise

- Difficult to interpret some.

4. Hybrid approaches ${ }^{10}$

\section{Orthodontic Index}

Richmond et al. (1992) developed Peer Assessment Rating (PAR) in evaluating the orthodontic treatment outcome. It sums up the data about misalignment and deviation from the ideal occlusion. The success is assessed by comparing the pre and post-treatment PAR scores. ${ }^{14}$

Zarei et al. (2006) developed ANN to predict the treatment outcomes in Class II and Class III patients. The results of the study concluded that neural network model correctly predicted the final PAR score and linear regression. ${ }^{15}$

Kim et al. (2009) developed a system called feature wrapping to precisely predict the prognosis of Class III treatment. 16

\section{Artificial Intelligence in Treatment Planning \\ Craniofacial Growth Modification}

Lux et al. developed an artificial neural network called selforganizing neural maps in which the resultant growth data were classified and relationships of the various growth patterns monitored. It used the concept of tensor analysis for describing the craniofacial skeletal changes, with regard to their growth pattern. ${ }^{17}$ Kok et al. aimed to determine the cervical vertebrae stages (CVS) for growth and development by the commonly used seven artificial intelligence classifiers and compared the performance of these algorithms with each other. Genetic algorithms, expert systems, fuzzy logic, logistic regression, random forest, decision tree, $\mathrm{k}$ - nearest neighbor algorithm ( $\mathrm{k}-\mathrm{NN}$ ), support vector machine (SVM), Naive Bayes, and artificial neural networks are among the main artificial intelligence algorithms compared in the study. On an average ANN was the lost stable algorithm compared to the other mentioned logarithms. ${ }^{18}$ The automated $\mathrm{x}$ - rays' analysis was used in estimation of the skeletal age by hand wrist radiographs. Machine learning was applied to estimate the age and compared against the radiologists. It was used to determine the growth status of patients which was necessary for deciding whether to utilize the growth during treatment. ${ }^{5}$

\section{Management of Impacted Canine}

Impacted canine needs a complex therapeutic management requiring interdisciplinary approach for the final orthodontic and periodontal outcome. The treatment time gets extended depending on the displacement of canine in relation to the adjacent incisors. The Bayesian Network (BN) takes an intermediate approach between statistics and artificial intelligence. $^{2}$ Panoramic and lateral cephalometric radiographs are useful in predicting impacted maxillary canine based on the angular and linear measurements. The random forest algorithm proved to have highest accuracy and precisely anticipated the eruption status of canine (83\%). ${ }^{5}$ Wang et al. introduced a machine learning method called Learning-based multi-source IntegratioN frameworK for Segmentation (LINKS), which was used with CBCT to quantify the variation in maxilla in cases of unilateral canine impaction. ${ }^{19}$

\section{Extraction Demands}

The two major reasons for extraction of teeth are

1. Space to align the teeth in the presence of severe crowding

2. To allow the teeth to be moved (usually to retract incisors) so that the protrusion can be reduced, or the skeletal Class II or Class III problems can be camouflaged. ${ }^{2}$ Xie et al. built a decision-making expert system (ES) to predict whether extraction is required or not in patients aged between 11 and 15 years with malocclusion.

ANN utilizes the error backward propagation learning algorithm to reduce the chances of making the error. The study concluded that the neural network was $80 \%$ accurate in determining whether extraction or non - extraction treatment was required. 4

Jung et al. used the ANN to predict the detailed extraction patterns which showed $84 \%$ accuracy. Li P et al. utilized ANN 
to identify anchorage requirements in extraction cases and proved to be accurate $83 \%$ of the time. ${ }^{20}$

Kong et al. utilized an artificial neural network - multilayer perceptron to predict treatment plan, determine whether extraction or non - extraction is required, pattern of extraction and anchorage pattern. The results showed accuracy of $94 \%$ for extraction and non - extraction prediction, $84.2 \%$ for pattern of extraction and $92.8 \%$ for anchorage pattern, respectively. The most important features for prediction are crowding, upper arch, ANB and curve of Spee. Thereby concluding that the neural network can be used in providing guidance in treatment for the less experienced orthodontists. ${ }^{21}$

\section{Size Prediction}

During the mixed dentition period, it is important to predict the size of unerupted teeth in management of the developing occlusion of the growing children. Prediction helps in determining whether the space available is enough to allow eruption of permanent teeth and for good alignment. The three commonly used methods for prediction are

1. Radiographs

2. Mixed dentition analysis

3. Combination of radiographic and non-radiographic method.

Moghimi et al. (2012) predicted the size of unerupted teeth for treatment planning. He used the neural network - hybrid system and genetic algorithms to predict the size of canine and premolar. The study concluded that the neural network is a promising tool for predicting the sizes of unerupted canines and premolars with greater accuracy than linear regression analyses. The structure of the technique can be adjusted based on the data collected from different ethnic groups. ${ }^{22}$

\section{In Treatment outcome}

\section{Head Gear}

Headgear, an orthopaedic device generates extraoral forces to the upper arch for anchorage, distalization or to inhibit forward maxillary growth. There are three types - high, medium, and low pull headgears. Akgam et al. developed a computer assisted inference model for selecting the right type of headgear appliance that acted as a decision-making aid for less experienced orthodontists. The model was designed to calculate the degree of certainty for choosing low, medium or high pull types of headgear. Eight orthodontic experts evaluated the decisions inferred by the system. The system correctly identified the precise headgears most of the time. ${ }^{23}$

\section{Soft Tissue Outcome}

An essential part of orthodontic diagnosis and treatment plan is the evaluation of facial profile. Facial soft tissue characteristics includes facial balance and harmony. The soft tissue aesthetics adds dimension to the bare skeleton. The relationship of nose, lips and chin is essential in obtaining an aesthetic dimension in the orthodontic treatment.

Nanda SB et al. proved that ANN could predict the changes in the lip curvature following orthodontic treatment with or without extractions. The predicted change and actual change was $29.6 \%$ and $7 \%$ for upper and lower lip respectively. 24

Patcas $\mathrm{R}$ et al. used an ANN to quantify the facial attractiveness pre- and post-orthognathic surgery on a scale from 0 to 100 . The results of the study concluded that the scores obtained before and after surgery were significant with improvement in facial attractiveness. ${ }^{25}$

Force System Prediction Using AI

As a result of the applied force and the tissue response, tooth movement and orthopaedic changes occur. In orthodontics, force system plays a major role to attain desirable tooth movement.

Kazem et al. constructed an artificial neural network to evaluate the force system of $\mathrm{T}$ - retraction springs and found that neural network was effective in input - output mapping. ${ }^{26}$

\section{CONCLUSIONS}

The field of artificial intelligence (AI) has grown remarkably in the medical field. The advances in AI like neural networking, natural language processing, image recognition and speech recognition have renovated the field of medicine and dentistry. The advantages of AI are efficiency, accuracy, precision, decreased workload, time saving and better monitoring.

AI incorporated are useful in simulation of the clinical situations under the following - diagnosis, treatment planning and treatment. It is particularly important in the digital era of dentistry, where all the records of the patient are being digitalized. AI should be applied only after careful considerations, when used incorrectly it can provide misleading information. So, humans should have a basic understanding on AI models to assist in clinical judgement and not to replace the knowledge and expertise of humans.

Financial or other competing interests: None.

Disclosure forms provided by the authors are available with the full text of this article at jemds.com.

\section{REFERENCES}

[1] Kunz F, Stellzig-Eisenhauer A, Zeman F, et al. Artificial intelligence in orthodontics: evaluation of a fully automated cephalometric analysis using a customized convolutional neural network. J Orofac Orthop 2020;81(1):52-68.

[2] Bahaa K, Noor G, Yousif Y. The artificial intelligence approach for diagnosis, treatment and modelling in orthodontic. Principles in Contemporary Orthodontics 2011:451.

[3] Martina R, Teti R, D'Addona GI, et al. Neural networkbased system for decision making support in orthodontic extractions. Intelligent Production Machines and systems 2006:235-40.

[4] Xie X, Wang L, Wang A. Artificial neural network modeling for deciding if extractions are necessary prior to orthodontic treatment. Angle Orthod 2010;80(2):262-6.

[5] Asiri SN, Tadlock LP, Schneiderman E, et al. Applications of artificial intelligence and machine learning in orthodontics. APOS Trends in Orthodontics 2020;10(1):17-24. 
[6] Poedjiastoeti W, Suebnukarn S. Application of convolutional neural network in the diagnosis of jaw tumors. Healthc inform Res 2018;24(3):236-41.

[7] Yong TH, Lee SJ, Yi WJ. Odontogenic cysts and tumors detection in panoramic radiographs using Deep Convolutional Neural Network (DCNN). International Conference on Medical Imaging with Deep Learning 2019.

[8] Yilmaz E, Kayikcioglu T, Kayipmaz S. Computer-aided diagnosis of periapical cyst and keratocystic odontogenic tumor on cone beam computed tomography. Comput Methods Programs Biomed 2017;146:91-100.

[9] Park JH, Hwang HW, Moon JH, et al. Automated identification of cephalometric landmarks: part 1comparisons between the latest deep-learning methods YOLOV3 and SSD. Angle Orthod 2019;89(6):903-9.

[10] Leonardi R, Giordano D, Maiorana F, et al. Automatic cephalometric analysis. Angle Orthod 2008;78(1):14551.

[11] Arik SÖ, Ibragimov B, Xing L. Fully automated quantitative cephalometry using convolutional neural networks. J Med Imaging (Bellingham) 2017;4(1):014501.

[12] Lee KS, Ryu JJ, Jang HS, et al. Deep convolutional neural networks based analysis of cephalometric radiographs for differential diagnosis of orthognathic surgery indications. Appl Sci 2020;10(6):2124.

[13] Hwang HW, Park JH, Moon JH, et al. Automated identification of cephalometric landmarks: part 2-might it be better than human? Angle Orthod 2020;90(1):69-76.

[14] Richmond S, Shaw WC, O'brien KD, et al. The development of the PAR index (Peer Assessment Rating): reliability and validity. Eur J Orthod 1992;14(2):125-39.

[15] Zarei A, El-Sharkawi M, Hairfield M, et al. An intelligent system for prediction of orthodontic treatment outcome. Proceedings of the IEEE International Joint Conference on Neural Network 2006: p. 2702-6.

[16] Kim BM, Kang BY, Kim HG, et al. Prognosis prediction for class III malocclusion treatment by feature wrapping method. Angle Orthod 2009;79(4):683-91.
[17] Lux CJ, Stellzig A, Volz D, et al. A neural network approach to the analysis and classification of human craniofacial growth. Growth Dev Aging1998;62(3):95-106.

[18] Kök H, Acilar AM, İzgi MS. Usage and comparison of artificial intelligence algorithms for determination of growth and development by cervical vertebrae stages in orthodontics. Prog Orthod 2019;20(1):41.

[19] Chen S, Wang L, Li G, et al. Machine learning in orthodontics: Introducing a 3D auto-segmentation and auto-landmark finder of CBCT images to assess maxillary constriction in unilateral impacted canine patients. Angle Orthod 2020;90(1):77-84.

[20] Jung SK, Kim TW. New approach for the diagnosis of extractions with neural network machine learning. Am J Orthod Dentofacial Orthop 2016;149(1):127-33.

[21] Li P, Kong D, Tang T, et al. Orthodontic treatment planning based on artificial neural networks. Scientific Reports 2019;9(1):1-9.

[22] Moghimi S, Talebi M, Parisay I. Design and implementation of a hybrid genetic algorithm and artificial neural network system for predicting the sizes of unerupted canines and premolars. Eur J Orthod 2012;34(4):480-6.

[23] Akçam MO, Takada K. Fuzzy modelling for selecting headgear types. Eur J Orthod 2002;24(1):99-106.

[24] Nanda SB, Kalha AS, Jena AK, et al. Artificial neural network (ANN) modeling and analysis for the prediction of change in the lip curvature following extraction and non-extraction orthodontic treatment. J Dent Spec 2015;3(2):130-9.

[25] Patcas R, Bernini DA, Volokitin A, et al. Applying artificial intelligence to assess the impact of orthognathic treatment on facial attractiveness and estimated age. Int J Oral Maxillofac Surg 2019;48(1):77-83.

[26] Kazem BI, Ghaib NH, Grama NM. Experimental investigation and neural network modeling for force system of retraction t-spring for orthodontic treatment. Journal of Medical Devices 2010;4(2). 